TYP-2-DIABETES

\title{
Immer häufiger bei Jugendlichen
}

- Ein Typ-2-Diabetes ist im Kindes- und Jugendalter heute keine Seltenheit mehr. Bei dicken Kindern kann die Unterscheidung zwischen Typ 1 und Typ 2 schwierig sein. Beide Diabetesformen werden derzeit im Kindesalter immer häufiger beobachtet. Die Unterscheidung gelingt oft erst durch eine Bestimmung der Autoantikörper und ist wichtig aufgrund der therapeutischen Konsequenzen: Der Typ-1-Diabetes ist immer insulinpflichtig.

In den USA hat sich die Häufigkeit des Typ2-Diabetes im Kindes- und Jugendalter in den letzten zehn Jahren verzehnfacht, erklärte Prof. Silva Arslanian, Direktorin der Universitätskinderklinik in Pittsburgh. Die
Inzidenz beträgt 10,5/100 000 . Risikofaktoren sind Adipositas, ethnische Zugehörigkeit zu einer Minderheit wie Indianer, Farbige oder Hispanics, eine positive Familienanamnese für Typ-2-Diabetes, $\mathrm{Pu}$ bertät und weibliches Geschlecht. Auch beim Syndrom

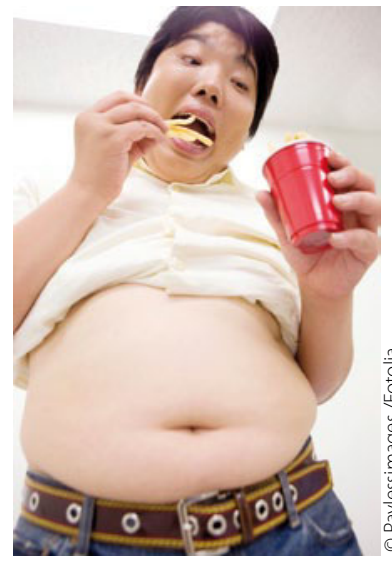

Der Typ-2-Diabetes bei Kindern hat sich verzehnfacht.

Diabetes vor. In den USA ist dies bei $8-45 \%$ der Patienten der Fall.

Die Behandlung des Typ2-Diabetes im Kindesalter kann problematisch sein, weil viele Medikamente für Kinder nicht zugelassen sind. Die Basistherapie besteht aus Metformin oder bei Acanthosis nigrans ist das Typ-2Diabetes-Risiko im Jugendalter erhöht. In Europa ist das Problem noch deutlich seltener, wenngleich ebenfalls ansteigend. Nur bei $0,16 \%$ bis $7 \%$ aller Diabetiker im Kindes- und Jugendalter liegt ein Typ-2- zusammen mit Lebensstilmodifikationen, Sport und Ernährungsumstellung.

DE

Jahreskongress der European Association for the Study of Diabetes, Lissabon, September 2011

\section{Burnout: Grundgefühl Unzufriedenheit}

Nachdem sich der Bundesliga-Trainer Ralf Rangnick zu einem Burnout bekannt hat, ist das Thema in aller Munde. Wir haben den Arzt und Burnout-Coach Dr. Thomas Bergner dazu befragt.

MMW: Wird das Burnout-Syndrom langsam zu einer Art Modeerkrankung oder ist es tatsächlich ein Phänomen, das immer mehr um sich greift?

Bergner: Es deutet viel darauf hin, dass die Zahl der Burnout-Kranken tatsächlich zunimmt. Ob sie so stark zunimmt wie die Zahl der Medienberichte darüber, das darf bezweifelt werden. Insofern hat es auch

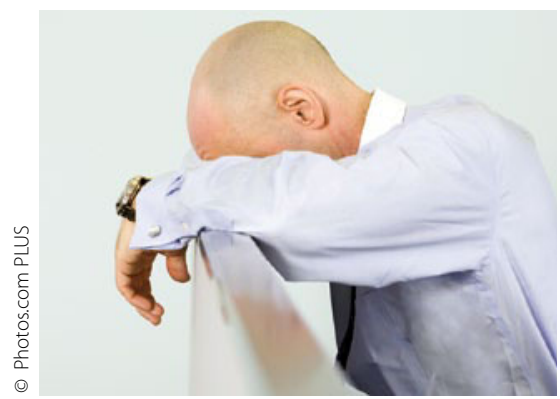

„Ich kann nicht mehr. Was tu ich hier eigentlich?" einen modischen Charakter, und man muss schauen, dass nicht jede Depression, jede Sucht- und jede Angsterkrankung in diesen Topf geworfen wird. Der Vorteil des Burnouts aus Sicht des Betroffenen ist, dass es als psychische Diagnose wahrgenommen wird, bei der man sich nicht schämen muss. Dagegen schämt sich jeder mit einer Suchtoder Angsterkrankung. Man muss also sehr genau schauen, ob jemand tatsächlich Burnout oder etwas anderes hat.

MMW: Wie kann man dies unterscheiden?

Bergner: Indem man die typische Symptomtrias bei Burnout abfragt. Da ist zum einen die emotionale Erschöpfung. Übliche Sätze sind "Ich kann nicht mehr. Was tu ich hier eigentlich?" Fragt man sich das nicht nur einmal im Monat, sondern ständig, und dominieren solche Gedanken und Gefühle das Leben, dann ist man emotional erschöpft. Das zweite Symptom ist der gesellschaftliche Rückzug: Man gibt Kontakte, Ehrenämter oder Vereinstätigkeiten auf, versucht sich in sein Schneckenhaus zurückzuziehen. Schließlich kommt es als

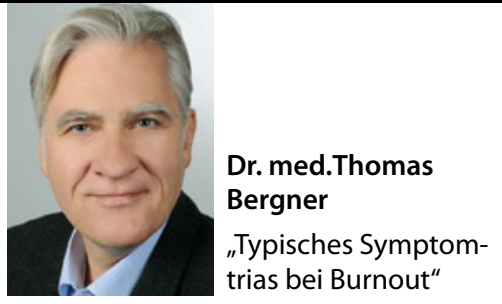

drittes zur Leistungsabnahme. Diese muss nicht offen erkennbar sein. Die Frage ist hier vielmehr, ob die Betroffenen mehr Willenseinsatz brauchen, ob sie sich mehr dazu zwingen müssen, um die übliche Leistung zu bringen.

MMW: Auch bei Depressiven würde man ein solches Verhalten erwarten.

Bergner: Richtig. Das ist sehr schwer zu unterscheiden. Es gibt aber beim Grundgefühl einen Unterschied: Bei Burnout ist dies nicht Traurigkeit, sondern Unzufriedenheit, und zwar schon dann, wenn es den Betroffenen nach außen hin noch richtig gut geht. Diese wird oft auf den Beruf projiziert. Tatsächlich steckt dahinter vielleicht etwas ganz anderes, ein Problem, das aus der Kindheit mitgeschleppt wird, ein Partnerschaftsproblem, finanzielle Schwierigkeiten oder eine Kombination davon.

INTERVIEW: THOMAS MÜLLER 